\title{
Elevated circulating stearic acid leads to a major lipotoxic effect on mouse pancreatic beta cells in hyperlipidaemia via a miR-34a-5p-mediated PERK/p53-dependent pathway
}

\author{
Huimin $\mathrm{Lu}^{1} \cdot$ Liuyi Hao ${ }^{1} \cdot$ Songtao $\mathrm{Li}^{1} \cdot \operatorname{Song} \mathrm{Lin}^{1} \cdot \operatorname{Lin} \mathrm{Lv}^{1} \cdot \operatorname{Yang} \mathrm{Chen}^{1} \cdot$ \\ Hongli Cui ${ }^{1}$ Tianqi $\mathrm{Zi}^{1} \cdot \mathrm{Xia} \mathrm{Chu}^{1} \cdot$ Lixin Na ${ }^{1}$ Changhao Sun ${ }^{1,2}$
}

Received: 8 August 2015 / Accepted: 25 January 2016/Published online: 11 March 2016

(C) Springer-Verlag Berlin Heidelberg 2016

\begin{abstract}
Aims/hypothesis Serum stearic acid (C18:0) is elevated in individuals with hyperlipidaemia and type 2 diabetes. However, the lipotoxicity induced by increased stearic acid in beta cells has not been well described. This study aimed to examine the adverse effects of stearic acid on beta cells and the potential mechanisms through which these are mediated.

Methods Three groups of C57BL/6 mice were fed a normal diet or a high-stearic-acid/high-palmitic-acid diet for 24 weeks, respectively. The microRNA (miR) profiles of islets were determined by microarray screening. Islet injury was detected with co-staining using the TUNEL assay and insulin labelling. A lentiviral vector expressing anti-miRNA-34a-5p oligonucleotide (AMO-34a-5p) was injected into mice via an intraductal pancreatic route.

Results In both mouse islets and cultured rat insulinoma INS1 cells, stearic acid exhibited a stronger lipotoxic role than
\end{abstract}

Huimin Lu, Liuyi Hao and Songtao Li contributed equally to this work

Electronic supplementary material The online version of this article (doi:10.1007/s00125-016-3900-0) contains peer-reviewed but unedited supplementary material, which is available to authorised users.

Lixin $\mathrm{Na}$

nalixin2003@163.com

$\triangle$ Changhao Sun

changhaosun2002@163.com

Department of Nutrition and Food Hygiene, Public Health College, Harbin Medical University, 157 Baojian Road, Nangang District, Harbin, Hei Longjiang Province 150081, People's Republic of China

2 Research Institute of Food, Nutrition and Health, Sino-Russian Medical Research Center, Harbin Medical University, Harbin, People's Republic of China other fatty acids, owing to repression of B cell CLL/ lymphoma 2 (BCL-2) and BCL-2-like 2 (BCL-W) by stearic acid stimulation of miR-34a-5p. The stearic-acid-induced lipotoxicity and reduction in insulin secretion were alleviated by AMO-34a-5p. Further investigations in INS-1 cells revealed that $\mathrm{p} 53$ was involved in stearic-acid-induced elevation of miR-34a-5p, owing in part to activation of protein kinaselike endoplasmic reticulum kinase (PERK). Conversely, silencing PERK alleviated stearic-acid-induced p53, miR-34a$5 \mathrm{p}$ and lipotoxicity.

Conclusions/interpretation These findings provide new insight for understanding the molecular mechanisms underlying not only the deleterious impact of stearic-acid-induced lipotoxicity but also apoptosis in beta cells and progression to type 2 diabetes.

Keywords Lipotoxicity $\cdot$ MicroRNA $\cdot$ Pancreatic beta cells . Stearic acid

$\begin{array}{ll}\begin{array}{l}\text { Abbreviations } \\ \text { AMO-34a-5p }\end{array} & \text { Anti-miRNA-34a-5p oligonucleotide } \\ \text { ATF6 } & \text { Activating transcription factor 6 } \\ \text { BCL-2 } & \text { B cell CLL/lymphoma 2 } \\ \text { BCL-W } & \text { BCL2-like 2 } \\ \text { CHOP } & \text { CCAAT/enhancer binding protein } \\ & \text { homologous protein } \\ \text { ER } & \text { Endoplasmic reticulum } \\ \text { GSIS } & \text { Glucose-induced insulin secretion } \\ \text { HPD } & \text { High-palmitic-acid diet } \\ \text { HSD } & \text { High-stearic-acid diet } \\ \text { IRE1 } & \text { Inositol-requiring-enzyme 1 } \\ \text { Lenti-AMO-34a-5p } & \text { Lentiviral vector expressing } \\ & \text { anti-miRNA-34a-5p oligonucleotide } \\ \text { miRNA/miR } & \text { MicroRNA }\end{array}$




$\begin{array}{ll}\text { MTT } & \text { 3-(4,5-Dimethyl-2-thiazolyl)-2,5 } \\ & \text {-diphenyl-2H-tetrazolium bromide } \\ \text { PERK } & \begin{array}{l}\text { Protein kinase-like endoplasmic } \\ \text { reticulum kinase }\end{array} \\ \text { qPCR } & \text { Quantitative PCR } \\ \text { SFA } & \text { Saturated fatty acids }\end{array}$

\section{Introduction}

Lipotoxicity refers to the cellular dysfunction caused by an elevated level of NEFA. Accumulating evidence indicates that lipotoxicity contributes to beta cell injury and plays a critical role in the development of type 2 diabetes [1-3]. NEFA is chemically classified into saturated and unsaturated fatty acids. It is widely accepted that lipotoxicity is mostly induced by long-chain saturated fatty acids (SFA), such as palmitic (C16:0) and stearic acid (C18:0), whereas unsaturated fatty acids are, in general, less toxic, and may even be protective [4-6].

There is growing evidence that chronic consumption of a diet rich in saturated fats increases circulating SFA levels and the risk of type 2 diabetes [7, 8]. It has been observed that NEFA profiles in individuals with obesity or hyperlipidaemia are substantially altered compared with healthy individuals; such changes are regarded as an important risk factor for type 2 diabetes [7, 9]. Palmitic acid is widely employed as a representative fatty acid to investigate the relationship between SFA and type 2 diabetes because it accounts for the highest percentage of dietary NEFA for the human body. However, whether other alterations in NEFA profiles have a detrimental role in beta cell destruction and type 2 diabetes is unknown. Our previous study revealed that the profiles of both fasting and postprandial serum NEFA were significantly altered in patients with hyperlipidaemia [7]. Most strikingly, we observed that only the proportion of stearic acid in the serum increased dramatically in the postprandial state, which implies that patients with hyperlipidaemia have a considerably higher level of serum stearic acid most of the time. In animal models of insulin resistance, the ratio between palmitic and stearic acids was decreased from 3:1 in the normal group to $2: 1$ in mice fed a high-fat diet [7]. Research in vitro has clearly shown that increasing stearic acid stimulates severe lipotoxicity in hepatocytes and skeletal muscle cells $[5,10$, 11]. Added to our previous observation that an increased proportion of stearic acid in the serum is closely related to insulin resistance [7], we proposed that raising the proportion of stearic acid in the blood makes the greatest contribution to beta cell lipotoxicity relative to other NEFA, and plays a dominant role in type 2 diabetes progression. Moreover, we sought to explore the molecular mechanisms underlying the destructive effect of stearic acid on beta cells.
Although the molecular mechanisms of stearic-acidinduced lipotoxicity are not fully understood, it is evident that enhanced endoplasmic reticulum (ER) stress is a central contributor to lipotoxicity resulting from transcriptional reprogramming [12], activation of c-Jun N-terminal kinase [13], CCAAT/enhancer binding protein homologous protein (CHOP) [14], p53 and mitochondrial apoptosis pathways [15, 16]. Importantly, microRNAs (miRNAs), which are involved in post-transcriptional regulation under ER stress, have emerged as key regulators of hepatocyte and cardiac muscle lipotoxicity $[17,18]$. In islet beta cells, the involvement of miRNA in stearic-acid-mediated lipotoxicity is still unclear.

In this study, we hypothesised that increasing stearic acid induces more severe beta cell lipotoxicity than other NEFA and its underlying mechanism is related to miRNA (miR)$34 a-5 p$.

\section{Methods}

Materials Stock solutions of stearic and palmitic acids (Sigma, St Louis, MO, USA) were prepared as described previously [19]. The oligonucleotides and lentivirus are described in the electronic supplementary material (ESM) Methods.

Animal experiments All experimental procedures followed guidelines for the care and use of animals established by Harbin Medical University and approved by the Animal Experimentation Ethics Committee of Harbin Medical University. Seven-week-old male C57BL/6 mice were purchased from Vital River Laboratories (Beijing, China) and completely randomised to receive a normal diet, a highstearic-acid diet (HSD) or a high-palmitic-acid diet (HPD) (ESM Table 1). A lentiviral vector expressing anti-miRNA34a-5p oligonucleotide (AMO-34a-5p) (lenti-AMO-34a-5p) $\left(1 \times 10^{8} \mathrm{TU} / \mathrm{ml}\right)$ was slowly injected into mice via the pancreatic duct $(100 \mu \mathrm{l})$ after feeding for 24 weeks, as described previously [20]. Further measurements were performed at 4 weeks post-lentiviral infection. See ESM Methods. Experimenters were blind to group assignment and outcome assessment.

Serum NEFA profile and glucose and lipid measurement Blood samples were collected for further detection. See ESM Methods.

miRNA microarray analysis Oebiotech (Shanghai, China) performed the miRNA microarray work, processing 12 samples from mouse islets ( $n=4$ per group). See ESM Methods.

Co-staining using TUNEL assay and insulin-labelled islets Islet injury was detected with the In Situ Cell Death Detection Kit (TUNEL Fluorescence Kit, Roche, Indianapolis, IN, 
USA). Islets were labelled with anti-insulin antibody (Cell Signaling Technology, Minneapolis, MN, USA), and viewed by confocal microscopy. See ESM Methods.

Pancreatic insulin content The pancreas was extracted using the acid ethanol method as described previously [21], and the insulin level was detected.

Intravenous glucose tolerance tests IVGTTs were performed on overnight-fasted mice. For the measurement of insulin and glucose levels, blood samples were collected at $0,1,5,10,20,30$ and $60 \mathrm{~min}$ after glucose administration $(0.75 \mathrm{~g} / \mathrm{kg})$ to the tail vein.

Beta cell mass and histochemical analysis The pancreatic tissues were fixed, embedded in paraffin and stained with antiinsulin and anti-glucagon antibodies. See ESM Methods.

Insulin granules analysis Electron microscopy was employed to analyse the content of insulin granules in fixed pancreatic tissues as described previously [22].

Cell culture Rat insulinoma cell line INS-1 cells were cultured and treated with $600 \mu \mathrm{mol} / 1$ stearic or other fatty acids for $24 \mathrm{~h}$. Mouse pancreatic islets were isolated as described previously [23]. See ESM Methods. Experimenters were blind to group assignment and outcome assessment.

Cell viability measurements Cell viability was determined by assessment of lactate dehydrogenase (LDH) release, propidium iodide (PI) staining, 3-(4,5-dimethyl-2-thiazolyl)2,5-diphenyl-2H-tetrazolium bromide (MTT) assay and Hoechst staining. See ESM Methods.

Transfection procedures INS-1 cells were transfected with oligonucleotides or recombinant plasmid using Lipofectamine 2000 (Invitrogen), as described previously [24], and used in detection assays $48 \mathrm{~h}$ after transfection.

Luciferase activity assay The luciferase activity was detected using a luciferase activity assay. See ESM Methods.

Quantitative PCR The mRNA and miRNA levels were determined by quantitative PCR (qPCR). See ESM Methods. The primer sequences are shown in ESM Table 2.

Western blot The western blot procedure has been described previously [25]. See ESM Methods.

Insulin measurements Insulin levels in mouse serum and cell culture media were determined by mouse/rat insulin ELISA kit (Linco Research, St Charles, MO, USA). To assess glucose-induced insulin secretion (GSIS), cells were incubated in secretion buffer (see ESM Methods) for an additional $60 \mathrm{~min}$ with 2.8 or $20 \mathrm{mmol} / 1$ glucose.

Statistics Values are expressed as mean \pm SD. Multiple groups were analysed with one-way ANOVA followed by a Student-Newman-Keuls test. Two-group-only comparisons were carried out by $t$ test. A two-sided $p$ value $<0.05$ was considered statistically significant. Repeated-measures ANOVA was used to analyse the glucose and insulin levels during IVGTT.

\section{Results}

Stearic acid induces more severe lipotoxicity than palmitic acid As shown in ESM Fig. 1a, b, only stearic and palmitic acids had an obviously lipotoxic effect on tested cells. The dose-response curves for palmitic and stearic acids are shown in ESM Fig. 1c. A mouse model with different stearic/palmitic acid ratios was subsequently established to mimic and investigate the lipotoxicity of increased proportions of stearic acid in hyperlipidaemia. The model was successfully established, as evidenced by significant elevation of circulating stearic and palmitic acid levels in the HSD and HPD groups, respectively, compared with controls (ESM Table 3). Importantly, the palmitic acid:stearic acid ratio was lower than controls in the HSD group (1.7:1 vs 2.7:1, respectively) but higher in the HPD group (6.2:1) (ESM Fig. 2). Body weight, total cholesterol (TC), triacylglycerol (TG), and LDL-cholesterol (LDLC) were markedly higher in both HSD and HPD mice, whereas HDL-cholesterol (HDL-C) was significantly lower than in control mice (ESM Table 4). TUNEL results showed that pancreases from HSD mice contained more TUNEL-positive islets than pancreases from HPD mice, and beta cell mass was lower in the HSD group (Fig. 1a). Subsequently, the levels of cleaved PARP-1 and cleaved caspase-3 was increased in pancreatic islets (Fig. 1b). Further, the impaired glucose tolerance and second-phase insulin secretion in response to glucose were significantly enhanced in the HSD group compared with the HPD group (Fig. 1c, d). Also, immunohistochemical analysis of pancreatic tissue showed that the islets of HSD mice comprised more glucagon-positive cells than both normal mice and the HPD group (Fig. 1e). These data suggest that HSD feeding is more detrimental to islet cells than HPD feeding. We next incubated INS-1 cells in culture media with different ratios of palmitic/stearic acid for $24 \mathrm{~h}$ and measured cytotoxicity with Hoechst 33342 staining (Fig. 1f) and MTT assay (Fig. 1g). The results showed that lipotoxicity was enhanced in the presence of a higher proportion of stearic acid, and levels of cleaved PARP-1 and cleaved caspase- 3 proteins were elevated accordingly (Fig. 1h). Additionally, GSIS in stearic-acid-treated INS-1 supernatant fractions was dramatically lower than in palmitic-acid-treated cells (Fig. 1i). 

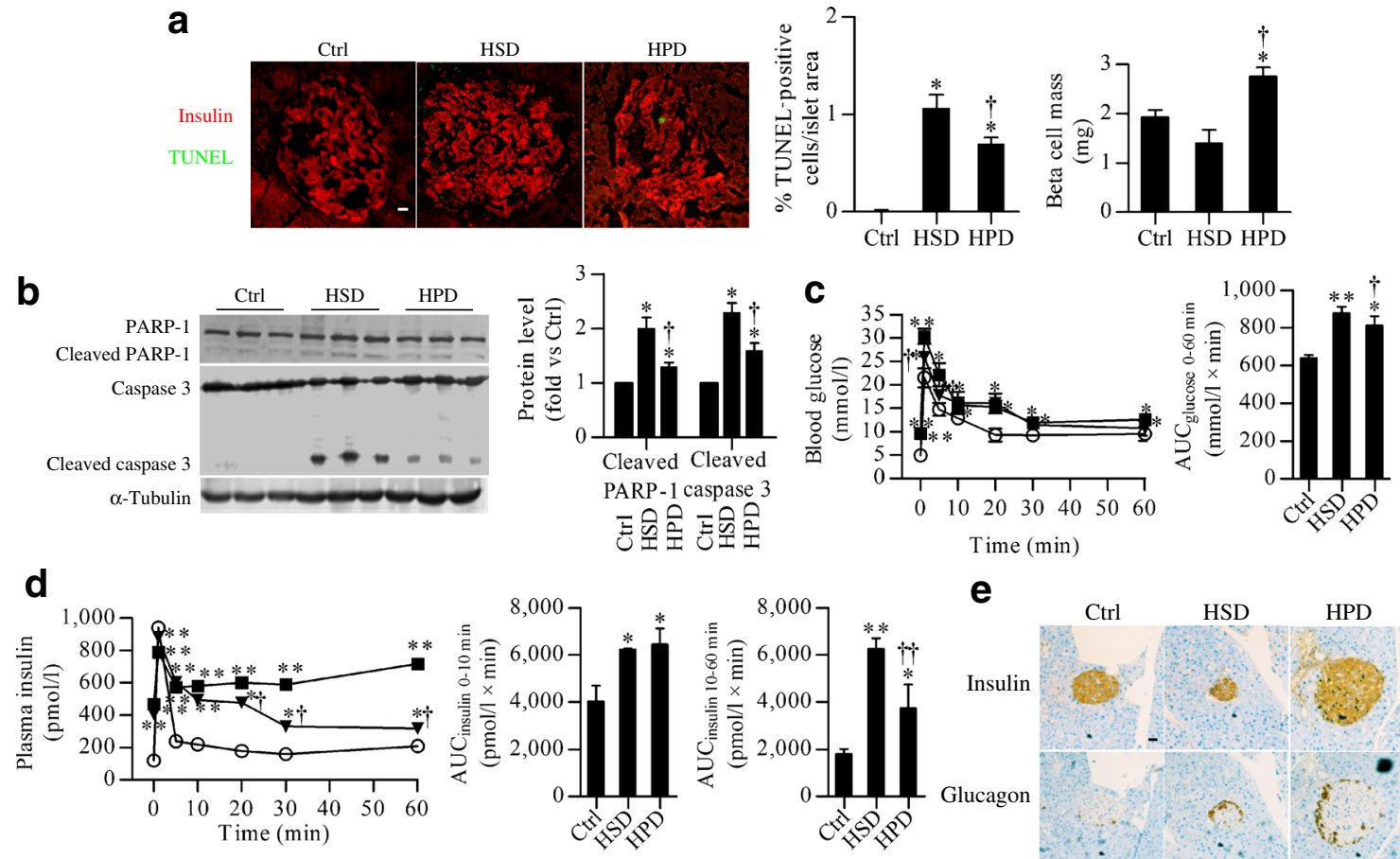

$$
\text { Time (min) }
$$

\section{e}
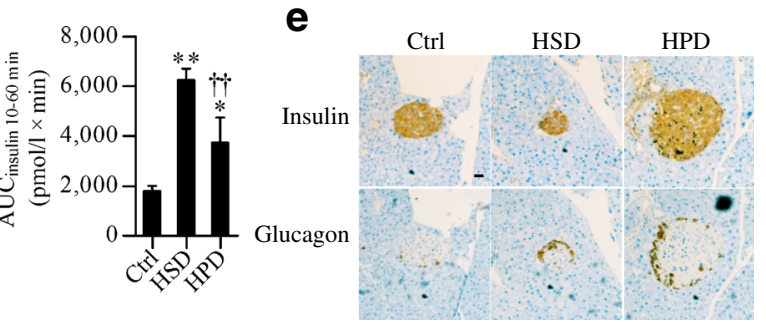

f
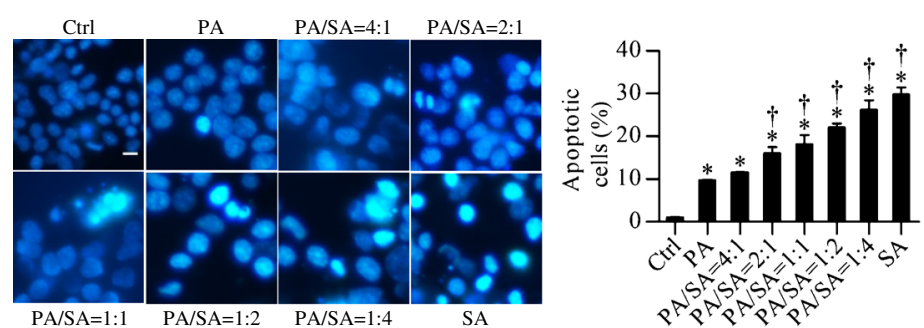

9

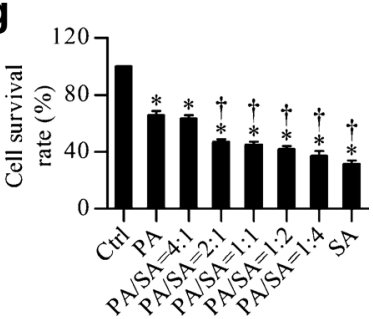

h
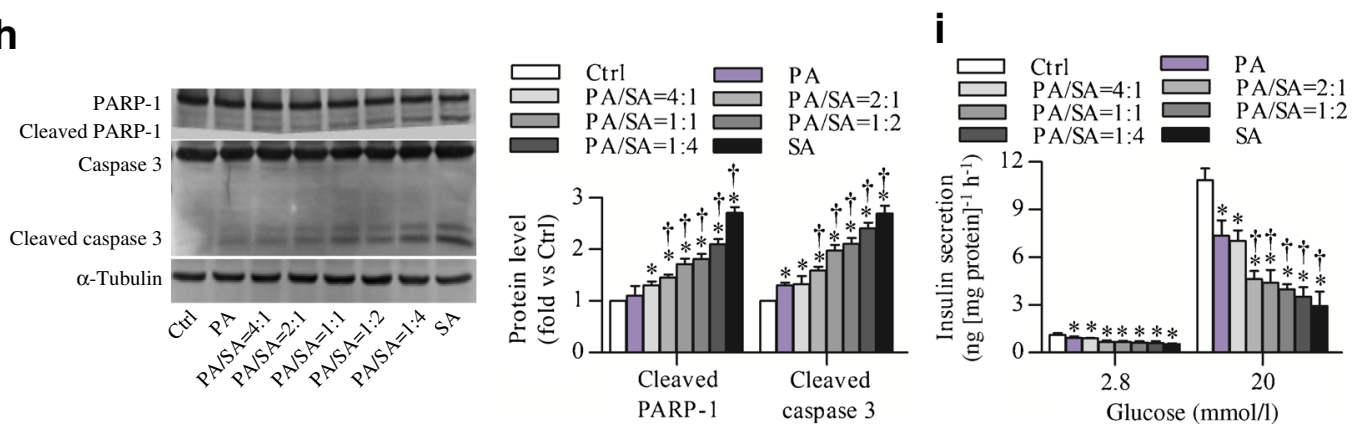

Fig. 1 Comparison of the lipotoxic effect of stearic acid (SA) and palmitic acid (PA) on islets and INS-1 cells. TUNEL staining (scale bar, $10 \mu \mathrm{m})(\mathbf{a})$, western blot analysis of cleaved PARP-1 and cleaved caspase 3 (b), blood glucose (c) and insulin (d) levels during IVGTT and immunohistochemical analysis of pancreas sections (scale bar, $100 \mu \mathrm{m})(\mathbf{e})$, from control, HSD and HPD mice; $n=6$ per group. In (a) islets are red and TUNEL-positive regions are green. In (c, d) circles, control; squares,

Stearic acid increases miR-34a-5p levels in both mouse islets and INS-1 cells In HSD islets, 40 miRNAs were differentially expressed (ten up- and 30 downregulated $\geq 2.0$-fold change and $p<0.05)$, whereas in HPD islets, 77 miRNAs were highly dysregulated (31 up- and 46 downregulated $\geq 2.0$-fold change and $p<0.05$ ), compared with normal mice
HSD; inverted triangles, HPD. $* p<0.05$ and $* * p<0.01$ vs control; ${ }^{\dagger} p<0.05$ and ${ }^{\dagger \dagger} p<0.01$ vs HSD. Lipotoxic effect of different PA:SA molar ratios (total concentration, $600 \mu \mathrm{mol} / \mathrm{l}$ ) on INS-1 cells measured by Hoechst staining (scale bar, $10 \mu \mathrm{m})(\mathbf{f})$, MTT assay (g), immunoblots of cleaved PARP-1/cleaved caspase 3 (h) and GSIS (i). ${ }^{*} p<0.05$ vs control; ${ }^{\dagger} p<0.05$ vs PA. The test was performed five times. Ctrl, control

(ESM Table 5). A heat map of HSD and HPD islet miRNA expression ratios ( $\log _{2}$ scale) is shown in ESM Fig. 3. Furthermore, we screened out six upregulated and 13 downregulated miRNAs that responded to an HSD, compared with both control and HPD groups $(\geq 2.0$ fold change and $p<0.05$, Fig. 2a). Among these, miR-34a-5p expression increased the 
Fig. 2 Differential expression of miRNAs in islets and INS-1 cells. (a) Heat maps and hierarchical clustering of expression ratios (log-log scale) of miRNAs in HSD mouse islets compared with both HPD and control mice. High relative expression is shown in red and low relative expression in green. $n=4$ per group. $(\mathbf{b}, \mathbf{c})$ qPCR verifies upregulation of miR-34a-5p in mice (b) and INS1 cells $(\mathbf{c}) .{ }^{*} p<0.05$ and $* * p<0.01$ vs controls; ${ }^{\dagger} p<0.05$ vs HSD or palmitic acid; $n=6$ per group; each in vitro test was performed five times. Ctrl, control; PA, palmitic acid; SA, stearic acid a

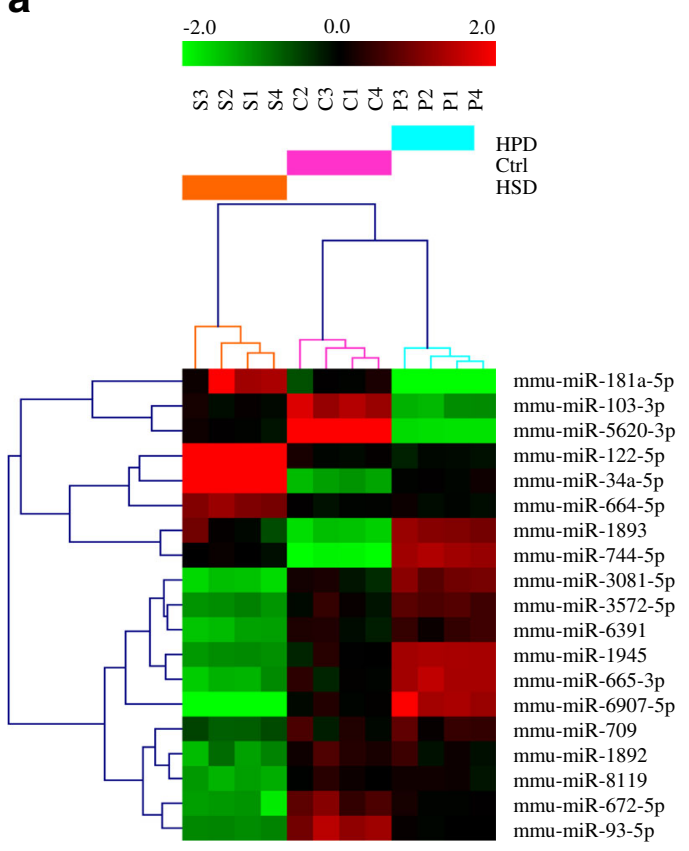

b

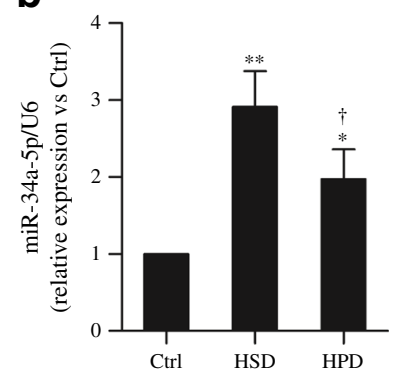

C

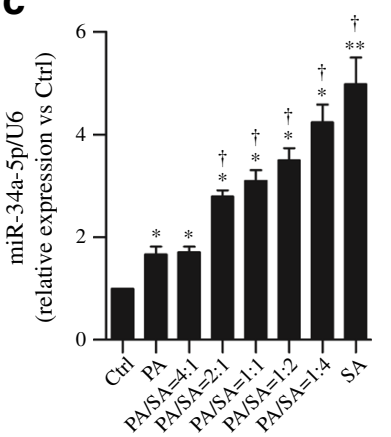

most, 6.35-fold in HSD and 1.57-fold in HPD mice, respectively.

Quantitative PCR verified that miR-34a-5p levels increased by $191 \%$ in HSD and $97 \%$ in HPD islets, respectively, compared with normal islets (Fig. 2b). In INS-1 cells exposed to different ratios of palmitic/stearic acids, miR-34a-5p expression was markedly upregulated compared with controls in cells exposed to a higher proportion of stearic acid, (Fig. 2c).

miR-34a-5p contributes to stearic-acid-induced lipotoxicity in islets and INS-1 cells Transfection of AMO$34 a-5 p$ into stearic-acid-treated INS- 1 cells efficiently reduced intracellular miR-34a-5p levels (ESM Fig. 4), inhibited stearic-acid-induced cell death (Fig. 3a, b), and reversed stearic-acid-increased cleaved PARP-1 and cleaved caspase3 levels (Fig. 3c). Accordingly, stearic-acid-decreased GSIS was apparently restored after miR-34a-5p inhibition (Fig. 3d). However, transfecting only miR-34a-5p into INS-1 cells also induced cell death and a reduction in GSIS, which were reversed by AMO-34a-5p (ESM Fig. 5a-d). In vivo, the lentiAMO-34a-5p was successfully delivered into the pancreas as evidenced by the strong green fluorescence (ESM Fig. 6a), with endogenous miR-34a-5p expression almost completely silenced (up to 95\%) in islets (ESM Fig. 6b). HSD-induced islet damage in mice was robustly alleviated by lenti-AMO$34 a-5 p$ injection, consistent with the decreased number of TUNEL-positive islets and increased beta cell mass (Fig. 3e), decreased levels of cleaved PARP-1 and cleaved caspase- 3 proteins (Fig. 3f) and improved glucose tolerance (Fig. $3 \mathrm{~g}$ ). Importantly, the first-phase insulin release was significantly enhanced and second-phase insulin secretion was decreased after lenti-AMO-34a-5p injection (Fig. 3h), which indicates that inhibition of miR-34a-5p could enhance the capacity to increase GSIS. Also, the number of alpha cells in the HSD + lenti-AMO-34a-5p group was significantly reduced and the normal cell distribution pattern was restored (Fig. 3i). Additionally, stearic-acid-mediated reductions in pancreatic insulin content (ESM Fig. 6c) and insulin granules (ESM Fig. 6d) were apparently reversed. The body weight and serum lipid level were not altered (ESM Table 6).

miR-34a-5p specifically represses BCL-2 and BCL-W expression Expression of B cell CLL/lymphoma 2 (BCL-2) and BCL-2-like 2 (BCL-W) was reduced in both HSD islets and stearic-acid-treated INS-1 cells (Fig. 4a, b). AMO-34a-5p treatment, either in vitro or in vivo, markedly reversed stearicacid-mediated downregulation of BCL-2 and BCL-W proteins, (Fig. 4c, d). In INS-1 cells overexpressing miR-34a-5p, levels of BCL-2 as well as BCL-W were decreased, but were efficiently rescued by addition of AMO-34a-5p (Fig. 4e). Furthermore, luciferase assay verified that overexpression of miR-34a-5p inhibited luciferase activity in human embryonic kidney 293 (HEK293) cells transfected with plasmid carrying the $3^{\prime}$ UTR of either the $B c l 2$ or the Bcl2l2 gene (Fig. $4 \mathrm{f}$ ).

p53 is involved in stearic acid-induced elevation of miR34a-5p The amount of $\mathrm{p} 53$ protein increased in HSD islets and stearic-acid-treated INS-1 cells (ESM Fig. 7a). Stearicacid-induced lipotoxicity was abrogated after silencing $p 53$ (also known as Trp53), accompanied by a 75\% reduction in stearic-acid-increased miR-34a-5p (Fig. 5a-c). Meanwhile, 
a

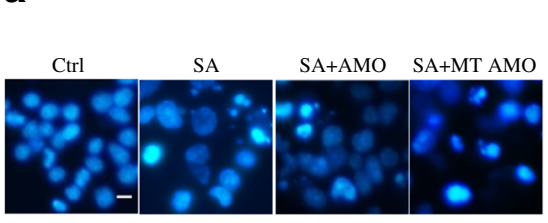

b

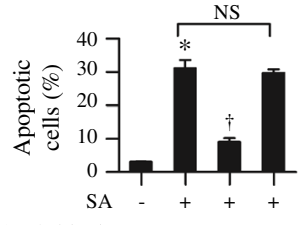

AMO-34a-5p

MT AMO-34a-5p

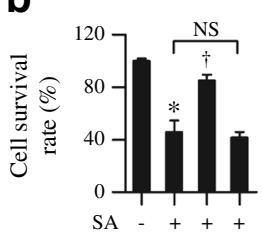

C
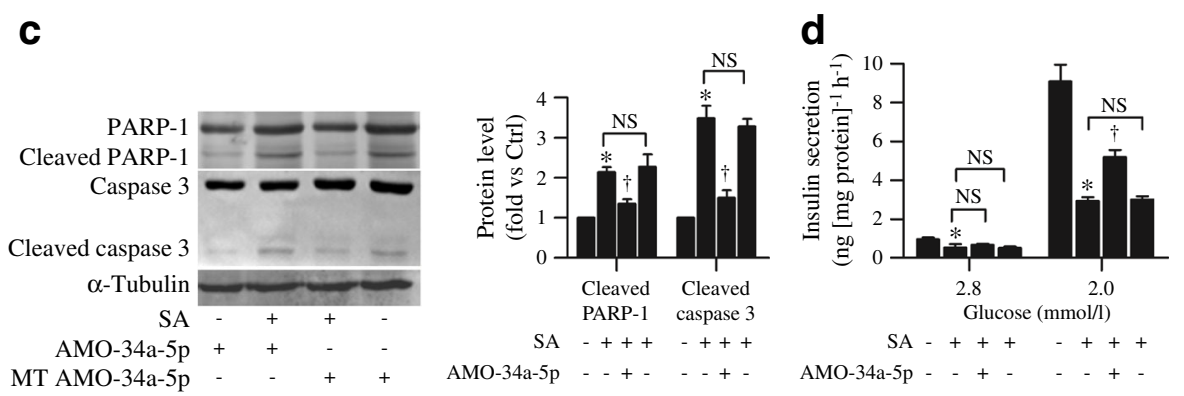

MT AMO-34a-5p - $\quad+\quad+$

$\begin{array}{rrr}\text { SA } & -+++ & -+++ \\ \text { AMO-34a-5p } & -++ & -\end{array}$

MT AMO-34a-5p

e
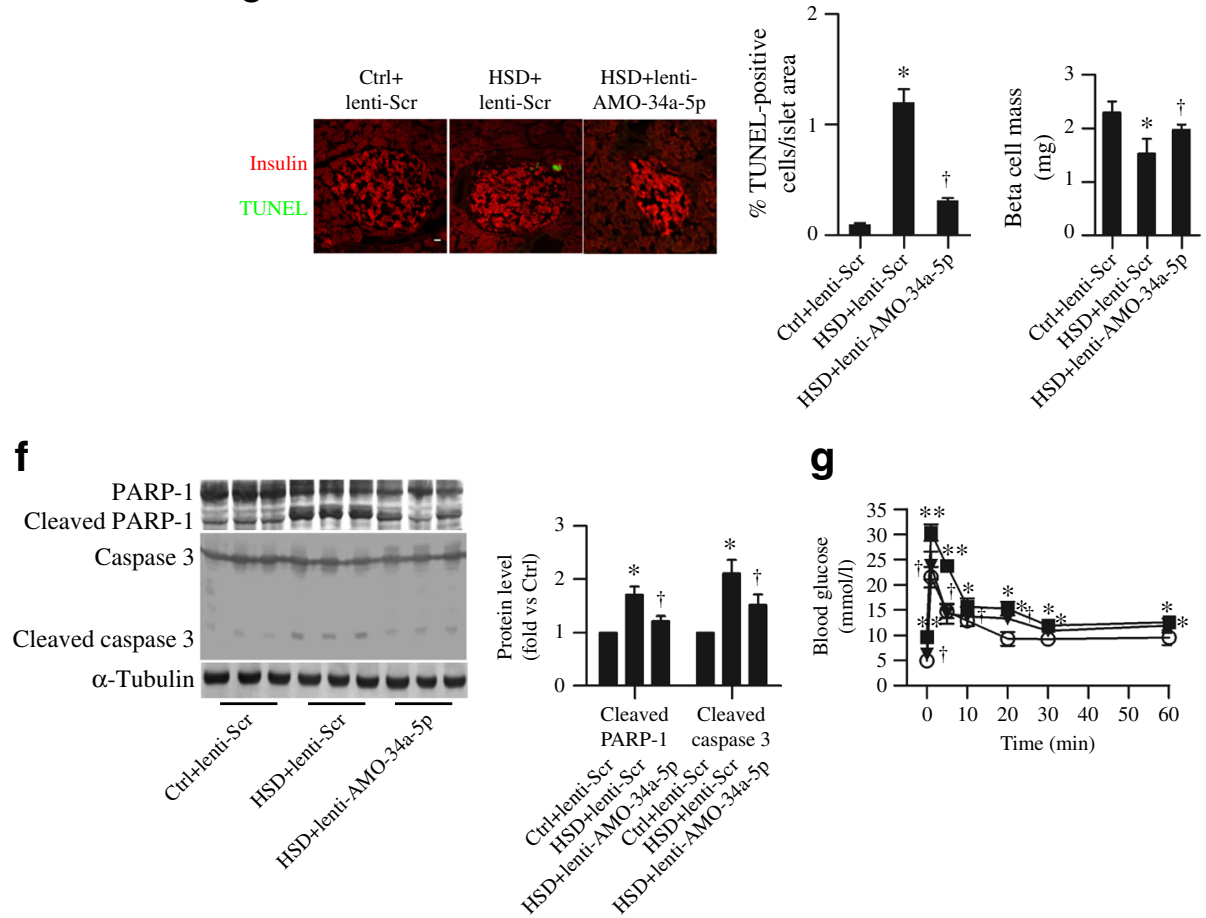

\section{g}

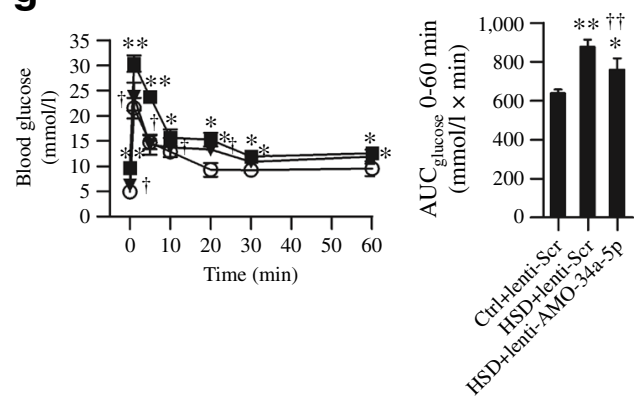

h
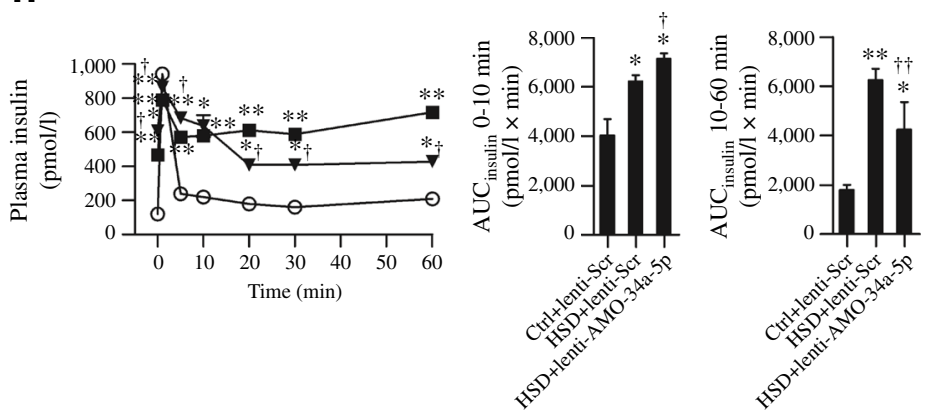

i

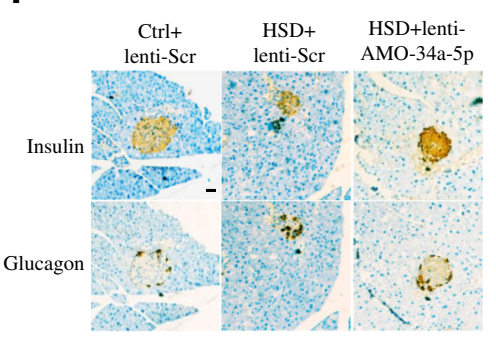


4 Fig. 3 Inhibition of miR-34a-5p improves stearic-acid-induced lipotoxicity in islets and INS-1 cells. Hoechst staining (scale bar, $10 \mu \mathrm{m})(\mathbf{a})$, MTT assay (b), immunoblotting of cleaved PARP-1/ cleaved caspase 3 (c) and GSIS (d) showing the effect of AMO-34a-5p on apoptosis in stearic acid-treated INS- 1 cells. ${ }^{*} p<0.05$ vs control; ${ }^{\dagger} p<0.05$ vs SA; each test was performed five times. TUNEL assay (e), western blot for cleaved PARP-1 cleaved caspase 3 (f), blood glucose (g) and insulin (h) level during IVGTT, and immunohistochemical analysis of pancreatic sections (scale bar, $100 \mu \mathrm{m}$ ) (i), showing the effect of lentiAMO-34a-5p on HSD islets. In (e) islets are red and TUNEL-positive regions are green (scale bar, $10 \mu \mathrm{m}$ ). Circles, control + lentiviral vector expressing scrambled AMO oligonucleotide (lenti-Scr); squares HSD + lenti-Scr; inverted triangles, HSD + lenti-AMO-34a-5p. ${ }^{*} p<0.05$ and ${ }^{* *} p<0.01$ vs control + lenti-Scr; ${ }^{\dagger} p<0.05$ and ${ }^{\dagger \dagger} p<0.01$ vs HSD + lenti-Scr. $n=6$ per group. AMO, anti-miRNA oligonucleotide; Ctrl, control; MT, mutant; PA, palmitic acid; SA, stearic acid

stearic-acid-elicited upregulation of cleaved PARP-1 and cleaved caspase-3, as well as the downregulation of BCL-2 and BCL-W protein expression, was rescued by 533 knockdown (Fig. 5d).

PERK contributes to stearic-acid-induced upregulation of miR-34a-5p and lipotoxicity Our in vitro results showed that the transmembrane sensors of ER stress, including inositolrequiring-enzyme 1 (IRE1), activating transcription factor 6 (ATF6) and PERK, are strongly upregulated by stearic acid treatment. Similarly, ER stress marker proteins, such as CHOP and glucose-regulated protein $78 \mathrm{kDa}$ (GRP78), were also enhanced (ESM Fig. 7b). Genetically silencing Perk (also known as Eif2ak3) by small interfering RNA (siRNA) in INS-1 cells significantly blocked the stearic-acid-induced increase in p53 expression (Fig. 6a), miR-34a-5p level (Fig. 6b) and lipotoxicity (Fig. 6c, d). Accordingly, stearic-acidincreased expression of cleaved PARP-1 and cleaved caspase 3 , as well as -suppressed BCL-2 and BCL-W, returned to basal levels after inhibition of Perk expression (Fig. 6e). However, gene silencing of neither Irel nor Atf6 inhibited the stearicacid-induced changes detected above (ESM Fig. 8). Further, the phosphorylated level of eukaryotic translation initiation factor (eIF) $2 \alpha$ was significantly enhanced after stearic acid exposure (ESM Fig. 9). The inhibitory efficiency of siRNAs targeting p53, Ire1, Atf6 and Perk are shown in ESM Fig. 10.

\section{Discussion}

In this study, we provide evidence that, relative to other NEFA, stearic acid is the major contributor to lipotoxicity in beta cells, implying that an increased concentration of serum stearic acid plays a fundamental role in the development of beta cell dysfunction and type 2 diabetes. Our mechanistic studies revealed that miR-34a-5p upregulation modulates stearic-acid-induced lipotoxicity by inhibiting the production of BCL-2 and BCL-W proteins. Additional studies have also a

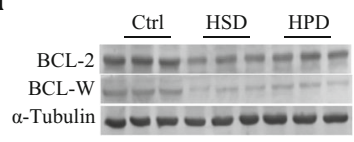

b

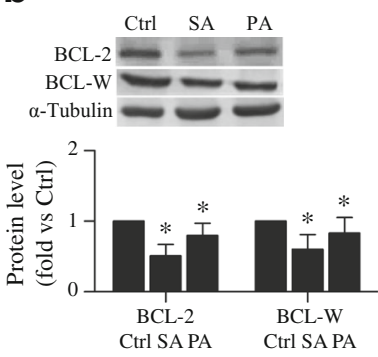

C
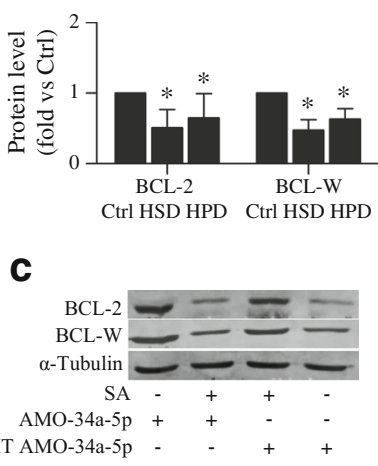

d
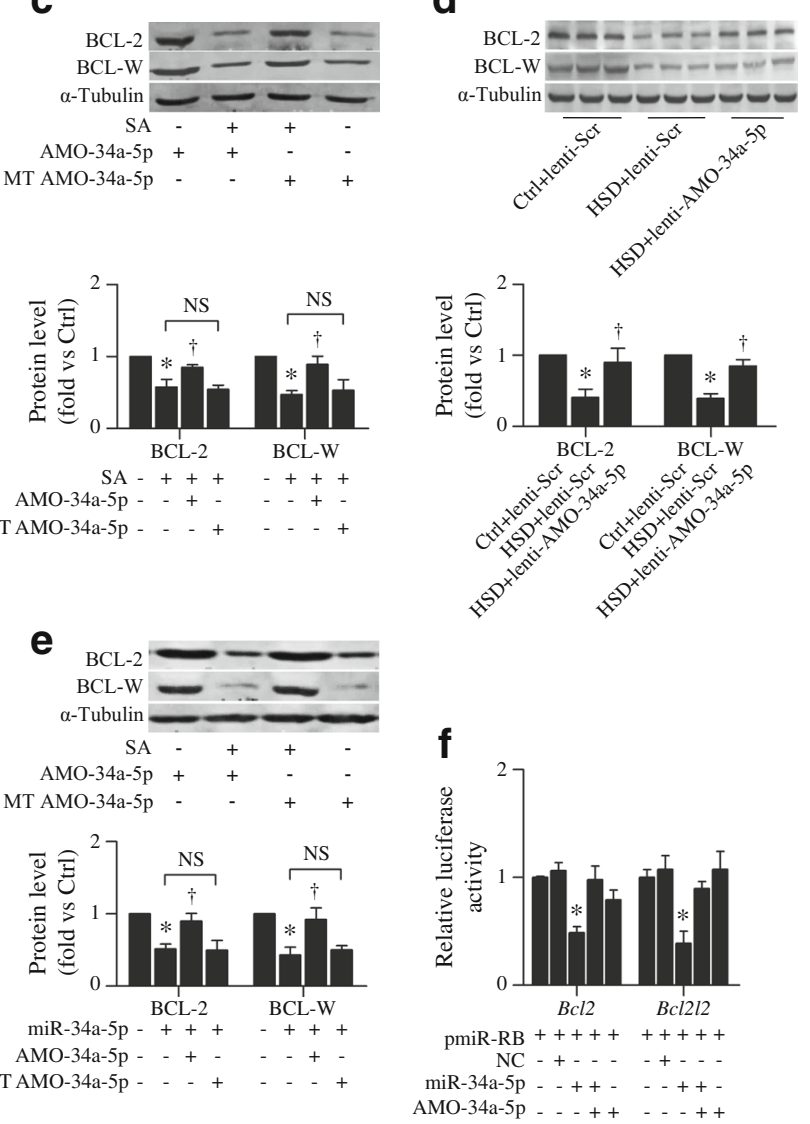

Fig. 4 Repression of $B c l 2$ and $B c l 2 l 2$ genes by miR-34a-5p. Downregulation of BCL-2/BCL-W in HSD islets (a) and stearic acidtreated INS-1 cells (b). Expression of BCL-2/BCL-W by AMO-34a-5p in SA-treated INS-1 cells (c) and HSD islets (d). (e) Effect of miR-34a-5p on BCL-2/BCL-W expression. (f) Effect of miR-34a-5p on the 3' UTR of $B c l 2$ and $B c l 2 l 2$ determined by luciferase activity assay. ${ }^{*} p<0.05$ vs control, ${ }^{\dagger} p<0.05$ for the indicated comparison. $n=6$ mice per group; each in vitro test was performed five times. AMO, anti-miRNA oligonucleotide; Ctrl, control; MT, mutant; PA, palmitic acid; pmiR-RB, pmiRRB REPORT; SA, stearic acid

demonstrated that the ER-stress-related sensor PERK contributes to stearic-acid-induced upregulation of miR-34a-5p in a p53-dependent signalling pathway.

Although alterations in the NEFA profiles of people with hyperlipidaemia have been widely investigated, the NEFA that is most directly associated with beta cell lipotoxicity remains unclear. Therefore, we first selected NEFA with 
Fig. 5 Involvement of p53 in stearic-acid-induced stimulation of miR-34a-5p in INS-1 cells. Effect of $p 53$ siRNA on miR-34a$5 \mathrm{p}$ levels (a), cell viability (b), apoptosis (scale bar, $10 \mu \mathrm{m})(\mathbf{c})$, and cleaved PARP-1/caspase 3/BCL-2/BCL-W protein expression (d) with/without stearic acid. ${ }^{*} p<0.05$ vs control; ${ }^{\dagger} p<0.05$ vs SA + siRNAnegative control; the test was performed five times. Ctrl, control; NC, negative control; SA, stearic acid a

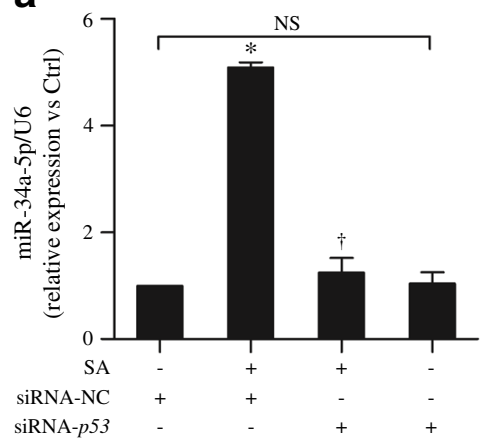

b

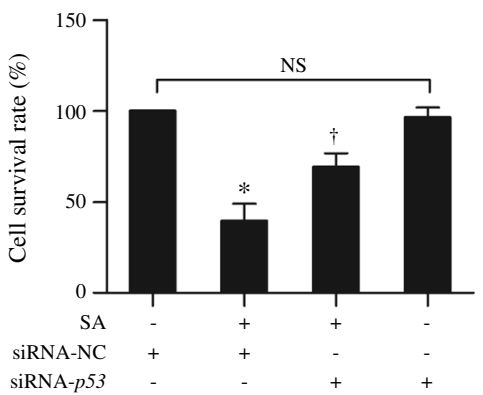

C
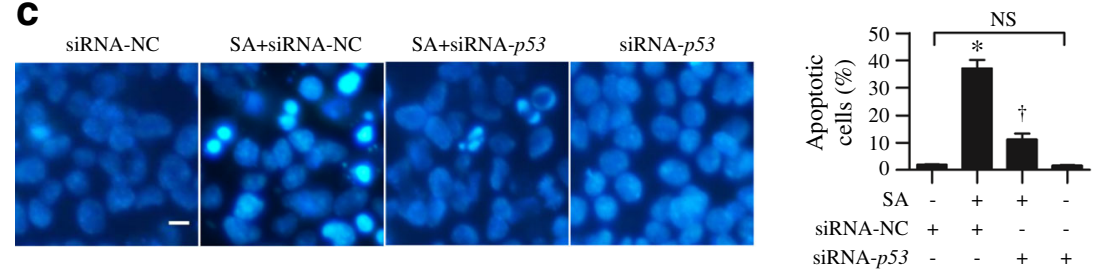

d

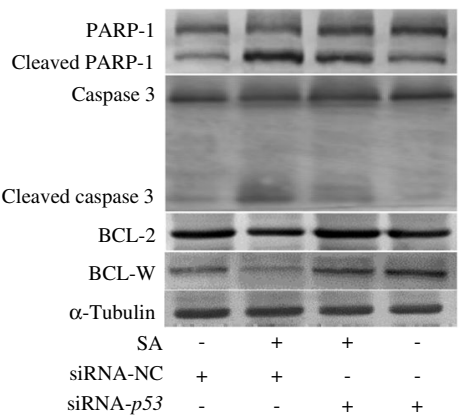

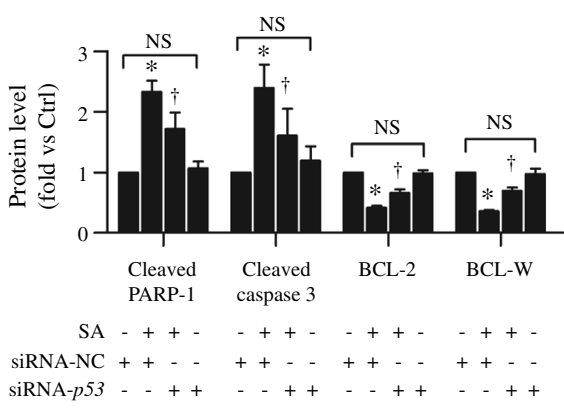

statistically discrepant levels between healthy and hyperlipidaemic individuals to test their cytotoxicity to pancreatic beta cells. We observed that only stearic and palmitic acids significantly lowered cell viability. Incubating cells in medium containing different ratios of palmitic acid:stearic acid dramatically enhanced cytotoxicity, but insulin secretion was reduced as the proportion of stearic acid was increased. The concentrations of palmitic and stearic acids used in this study were selected to avoid excessive cell death based on previous reports and our previous experiments [7, 26, 27], though this does not happen in human or animal pancreatic beta cells in vivo $[26,27]$. The concentrations of palmitic and stearic acids employed in cultured cells were, in fact, lower than in patients with hyperlipidaemia, but induced more severe damage than in the in vivo system, which could be explained by the existence of protective factors (i.e. unsaturated fatty acids [4-6]). Additionally, we observed that eicosapentaenoic acid (EPA; C20:5), a potential protective factor, was reduced in both HSD and HPD mice, and this might also contribute to high-fat-diet-induced lipotoxicity. Unlike the in vitro system, it is unrealistic to establish animal models with only stearic- or palmitic-acid-constructed lipid profiles in blood. However, a well-accepted approach to compare different biological function among the different types of NEFA was achieved by regulating the blood NEFA ratio using various oils as a fat source in the high-fat diet $[28,29]$. In this study, models of hyperlipidaemia with high circulating levels of either stearic or palmitic acid were established using lard or palm oil as a fat source, consistent with previous studies [28-30]. After 24 weeks of feeding, the fasting serum levels of stearic and palmitic acids increased in HSD and HPD mice, respectively. The palmitic acid:stearic acid ratio was 1.7:1 and 6.2:1 in HSD and HPD mice, respectively. Subsequently, the HSD mice showed significant impairment of glucose tolerance and GSIS compared with HPD mice, with an increased number of apoptotic beta cells leading to a decrease in beta cell mass. Our results clearly show that, in vivo, HSD induces more damage in islets than HPD, consistent with the in vitro results. No difference was observed in the total SFA content between the HSD and HPD groups, excluding the possibility that the different extent of lipotoxicity was caused by something other than differences in lipid concentrations. Additionally, these results are also consistent with our previous findings [7, 31], indicating that stearic acid appears to be a more important risk factor for type 2 diabetes than other NEFA. Therefore, great attention should be paid to elevated serum stearic acid, as it 
Fig. 6 Involvement of PERK in stearic acid-induced stimulation of miR-34a-5p via p53 in INS-1 cells. Effect of Perk siRNA on p53 protein expression (a), miR$34 a-5 p$ levels (b), cell survival (c), apoptosis (scale bar, $10 \mu \mathrm{m})(\mathbf{d})$, and cleaved PARP-1/cleaved caspase 3/BCL-2/BCL-W protein expression (e) with/without SA. ${ }^{*} p<0.05$ vs control; ${ }^{\dagger} p<0.05$ vs $\mathrm{SA}+$ siRNA-negative control; the test was performed five times. Ctrl, control; NC, negative control; SA, stearic acid a
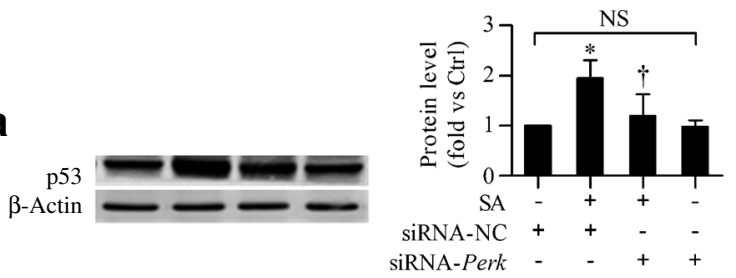

b

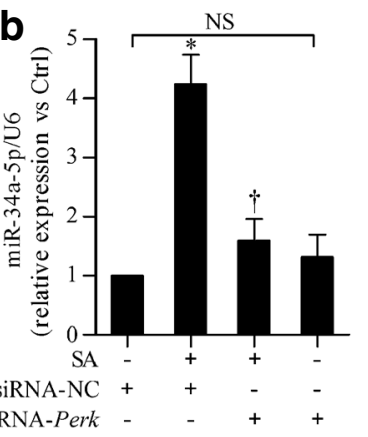

C

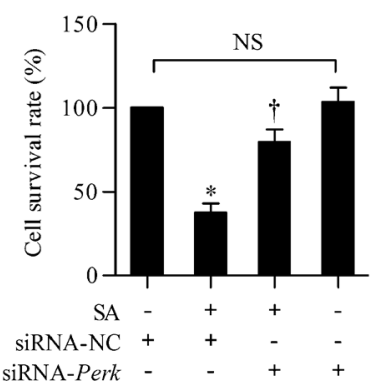

d
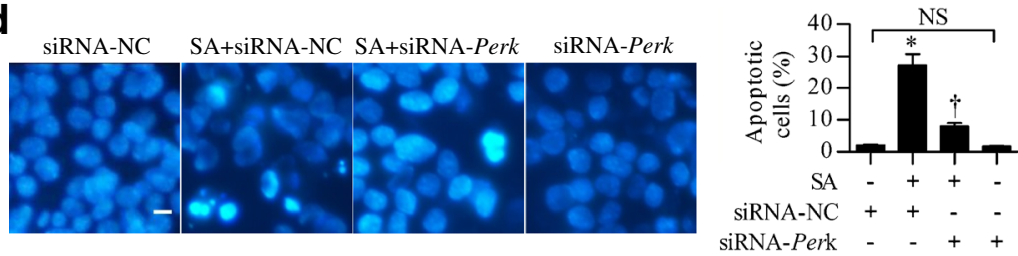

e
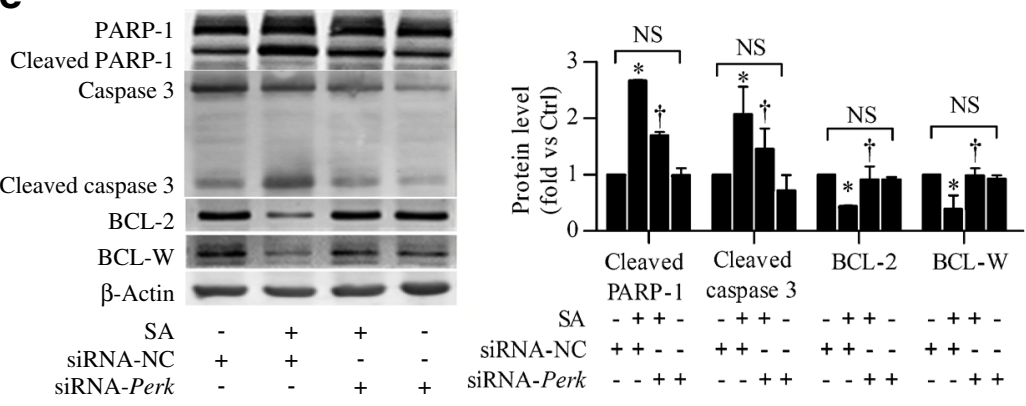

appears to have a greater negative impact on beta cell function.

The mechanistic pathways responsible for HSD-induced lipotoxicity in pancreatic beta cells are not fully understood. Recent evidence has provided new clues that miRNAs are involved in the regulation of beta cell failure and type 2 diabetes [32-34]. In our study, we selected 19 miRNAs that are differentially expressed in islets of HSD mice relative to HPD and normal mice, based on gene-chip microarray analysis. Among these miRNAs, miR-34a-5p displayed the largest fold increase, which was in line with previous observations that a high level of miR-34a-5p was present in the islets of diabetic $d b / d b$ mice and pancreatic islets exposed to palmitic acid [34]. To demonstrate the potential role of miR-34a-5p in HSDmediated lipotoxicity in beta cells, endogenous miR-34a-5p was abrogated by AMO-34a-5p in both mice and INS- 1 cells. Lenti-AMO-34a-5p injection ameliorated HSD-induced islet apoptosis in vivo. Accordingly, impaired glucose tolerance and GSIS in HSD mice were largely restored. The decrease in beta cell mass seen in HSD mice was also reversed. Insulin secretion and beta cell mass are two important factors for regulating glucose homeostasis through well-balanced beta cell compensation in the early stage of type 2 diabetes, but they gradually worsen $[35,36]$. Our results indicate that miR34a-5p inhibition in HSD islets could probably improve the beta cell dysfunction and further slow the progression of type 2 diabetes. In vitro, AMO-34a-5p relieved the cytotoxic effect of stearic acid and reversed the reduction in insulin secretion. Conversely, overexpression of miR-34a-5p significantly enhanced cytotoxicity and reduced GSIS. These results strongly suggest that miR-34a-5p plays a crucial role in elevating lipotoxicity induced by serum stearic acid. However, the stearic-acid-mediated decrease in GSIS was not completely reversed by miR-34a-5p inhibition, which implies that a non-miR-34a-5p-mediated mechanism operated in GSIS reduction. We also observed that miR-34a-5p levels were higher 
in HSD islets and stearic-acid-treated INS-1 cells than in HPD islets and the palmitic acid group, respectively, which suggests that the greater adverse effect of stearic over palmitic acid on beta cells, both in vivo and in vitro, is probably attributable to differential regulation of miR-34a-5p.

Previous studies have indicated that miR-34a-5p is closely associated with apoptosis through the BCL-2 family in cancer cells, tubular cells and cardiomyocytes [37-39]. The BCL-2 family includes both anti-apoptosis members (BCL-2, BCL2-like 1 [BCL-XL] and BCL-W) and pro-apoptosis members (BCL-2-associated X protein [BAX], BCL-2-antagonist/killer [BAK] and BCL-2-associated agonist of cell death [BAD]) $[40,41]$. In our study, we confirmed that BCL-2 and BCL$\mathrm{W}$ are direct targets of miR-34a-5p using luciferase activity assays. However, how stearic acid regulates miR-34a-5p levels is still unclear. Accumulating evidence supports p53 as a classic transcription factor regulating miR-34a-5p. To further investigate the involvement of p53 in miR-34a-5p upregulation after long-term incubation of INS-1 cells with stearic acid, we silenced $p 53$ and then investigated whether miR-34a-5p levels were reversed, with an associated reduction in lipotoxicity, which would suggest that the mechanism underlying HSD-induced stimulation of miR-34a-5p uses a p53-dependent pathway.

Enhanced ER stress, associated with beta cell lipotoxicity, transduces apoptotic signals in the early stage of type 2 diabetes [42]. Three major signalling pathways separately initiated by ER transmembrane sensor proteins, including IRE1, PERK and ATF6, are involved in ER stress [43]. We subsequently knocked down Ire1, Perk and Atf6 with siRNAs in stearicacid-treated INS-1 cells, and found that inhibition of Ire 1 and Atf6 failed to inhibit stearic-acid-induced stimulation of miR-34a-5p. However, transfection of siRNA for Perk attenuated lipotoxicity and miR-34a-5p levels on stearic acid treatment. These findings demonstrate that miR-34a-5p might be promoted by PERK in conditions of ER stress. We also discovered that PERK exhibited an inhibitory effect on p53 protein expression, suggesting that a PERK-p53-miR-34a-5p axis is mechanistically involved in stearic-acid-induced lipotoxicity in beta cells.

Additionally, the inflammatory response was the common reaction during prolonged exposure to NEFA, which in turn results in the aggravation of lipotoxicity. Chronic exposure to inflammatory conditions leads to beta cell dysfunction and apoptosis [32, 44, 45]. A recent investigation has revealed that the level of miR-34a increased concomitantly in cytokinetreated MIN6 cells and human pancreatic islets, and contributed to beta cell dysfunction [32]. Therefore, we proposed that the HSD-induced increase in miR-34a-5p and further pancreatic injury probably represented an important inflammatory response pathway. This needs to be clarified in future studies.

In conclusion, we provide evidence that elevated levels of circulating stearic acid induce severe lipotoxicity in pancreatic beta cells. It appears that upregulation of miR-34a-5p and the associated inhibition of BCL-2/BCL-W play a critical role in mediating stearic acid cytotoxicity. Our results also suggest a regulatory role for PERK in miR-34a-5p expression and that p53 is a mediator of PERK-induced miR-34a-5p upregulation. Collectively, these findings allow us to propose a novel signalling pathway linking stearic acid to lipotoxicity in beta cells: stearic acid-PERK-p53-miR-34a-5p-BCL-2/BCL-W. These findings provide a novel insight into stearic-acidinduced lipotoxicity and progression of type 2 diabetes, whereby lowering miR-34a-5p might be an effective strategy for improving lipotoxicity in beta cells.

Acknowledgements We thank B. X. Wu (Department of Nutrition and Food Hygiene, Public Health College, Harbin Medical University) for helping with serum glucose and lipid measurement and Q. N. Qu and Y. Y. Liu (Department of Nutrition and Food Hygiene, Public Health College, Harbin Medical University) for help with animal feeding.

Funding This work was supported by the State Key Program of National Natural Science of China (grant number 81130049), and the National Natural Science Foundation of China (grant numbers 81202187, 81202188 and 81202191).

Duality of interest The authors declare that there is no duality of interest associated with this manuscript.

Contribution statement CS, LN and SLi conceived the experiments, analysed and interpreted the data and reviewed and edited the manuscript. HL and SLi were responsible for designing and performing the study, acquisition and analysis of data and writing the draft of the manuscript. HL, LH, SLin, LL, YC, HC, TZ, XC and LN performed the study, collected data and interpreted and drafted the manuscript. All authors approved the final version of the manuscript. CS is the guarantor of this work.

\section{References}

1. Elsner M, Gehrmann W, Lenzen S (2011) Peroxisome-generated hydrogen peroxide as important mediator of lipotoxicity in insulinproducing cells. Diabetes 60:200-208

2. Giacca A, Xiao C, Oprescu AI, Carpentier AC, Lewis GF (2011) Lipid-induced pancreatic $\beta$-cell dysfunction: focus on in vivo studies. Am J Physiol Endocrinol Metab 300:E255-E262

3. Hodson DJ, Mitchell RK, Bellomo EA et al (2013) Lipotoxicity disrupts incretin-regulated human $\beta$ cell connectivity. J Clin Invest 123:4182-4194

4. Listenberger LL, Han X, Lewis SE et al (2003) Triglyceride accumulation protects against fatty acid-induced lipotoxicity. Proc Natl Acad Sci U S A 100:3077-3082

5. Zhang Y, Dong L, Yang X, Shi H, Zhang L (2011) $\alpha$-Linolenic acid prevents endoplasmic reticulum stress-mediated apoptosis of stearic acid lipotoxicity on primary rat hepatocytes. Lipids Health Dis 10:81

6. Cheon HG, Cho YS (2014) Protection of palmitic acid-mediated lipotoxicity by arachidonic acid via channeling of palmitic acid into triglycerides in C2C12. J Biomed Sci 21:13

7. Chu X, Liu L, Na L et al (2013) Sterol regulatory element-binding protein-1c mediates increase of postprandial stearic acid, a potential 
target for improving insulin resistance, in hyperlipidemia. Diabetes 62:561-571

8. Galbo T, Perry RJ, Jurczak MJ et al (2013) Saturated and unsaturated fat induce hepatic insulin resistance independently of TLR-4 signaling and ceramide synthesis in vivo. Proc Natl Acad Sci U S A 110:12780-12785

9. Hernandez TL, Sutherland JP, Wolfe P et al (2010) Lack of suppression of circulating free fatty acids and hypercholesterolemia during weight loss on a high-fat, low-carbohydrate diet. Am J Clin Nutr 91:578-585

10. Hirabara SM, Curi R, Maechler P (2010) Saturated fatty acidinduced insulin resistance is associated with mitochondrial dysfunction in skeletal muscle cells. J Cell Physiol 222:187-194

11. Sawada K, Kawabata K, Yamashita T, Kawasaki K, Yamamoto N, Ashida H (2012) Ameliorative effects of polyunsaturated fatty acids against palmitic acid-induced insulin resistance in L6 skeletal muscle cells. Lipids Health Dis 11:36

12. Zhang K (2015) Endoplasmic reticulum stress response and transcriptional reprogramming. Front Genet 5:460

13. Gan LT, Van Rooyen DM, Koina ME, McCuskey RS, Teoh NC, Farrell GC (2014) Hepatocyte free cholesterol lipotoxicity results from JNK1-mediated mitochondrial injury and is HMGB1 and TLR4-dependent. J Hepatol 61:1376-1384

14. Cazanave SC, Elmi NA, Akazawa Y, Bronk SF, Mott JL, Gores GJ (2010) CHOP and AP-1 cooperatively mediate PUMA expression during lipoapoptosis. Am J Physiol Gastrointest Liver Physiol 299:G236-G243

15. Kakisaka K, Cazanave SC, Fingas CD et al (2012) Mechanisms of lysophosphatidylcholine-induced hepatocyte lipoapoptosis. Am J Physiol Gastrointest Liver Physiol 302:G77-G84

16. Egnatchik RA, Leamy AK, Jacobson DA, Shiota M, Young JD (2014) ER calcium release promotes mitochondrial dysfunction and hepatic cell lipotoxicity in response to palmitate overload. Mol Metab 3:544-553

17. Miyamoto Y, Mauer AS, Kumar S, Mott JL, Malhi H (2014) MmumiR-615-3p regulates lipoapoptosis by inhibiting C/EBP homologous protein. PLoS One 9, e109637

18. Kuwabara Y, Horie T, Baba O et al (2015) MicroRNA-451 exacerbates lipotoxicity in cardiac myocytes and high-fat diet-induced cardiac hypertrophy in mice through suppression of the LKB1/AMPK pathway. Circ Res 116:279-288

19. Goldstein JL, Basu SK, Brown MS (1983) Receptor-mediated endocytosis of low-density lipoprotein in cultured cells. Methods Enzymol 98:241-260

20. Doiron B, Hu W, Norton L, DeFronzo RA (2012) Lentivirus shRNA Grb10 targeting the pancreas induces apoptosis and improved glucose tolerance due to decreased plasma glucagon levels. Diabetologia 55:719-728

21. Portha B, Picon L, Rosselin G (1979) Chemical diabetes in the adult rat as the spontaneous evolution of neonatal diabetes. Diabetologia 17:371-377

22. Wang H, Ishizaki R, Kobayashi E, Fujiwara T, Akagawa K, Izumi T (2011) Loss of granuphilin and loss of syntaxin-1A cause differential effects on insulin granule docking and fusion. J Biol Chem 286:32244-32250

23. Sutton R, Peters M, McShane P, Gray DW, Morris PJ (1986) Isolation of rat pancreatic islets by ductal injection of collagenase. Transplantation 42:689-691

24. Yang B, Lin H, Xiao J et al (2007) The muscle-specific microRNA miR-1 regulates cardiac arrhythmogenic potential by targeting GJA1 and KCNJ2. Nat Med 13:486-491

25. Lu N, Li Y, Qin H, Zhang YL, Sun CH (2008) Gossypin up-regulates LDL receptor through activation of ERK pathway: a signaling mechanism for the hypocholesterolemic effect. J Agric Food Chem 56:11526-11532

26. Thörn K, Bergsten P (2010) Fatty acid-induced oxidation and triglyceride formation is higher in insulin-producing MIN6 cells exposed to oleate compared to palmitate. J Cell Biochem 111:497-507

27. Litwak SA, Wali JA, Pappas EG et al (2015) Lipotoxic stress induces pancreatic $\beta$-cell apoptosis through modulation of $\mathrm{Bcl}-2$ proteins by the ubiquitin-proteasome system. J Diabetes Res 2015: 280615

28. van den Berg SA, Guigas B, Bijland S et al (2010) High levels of dietary stearate promote adiposity and deteriorate hepatic insulin sensitivity. Nutr Metab (Lond) 7:24

29. Tholstrup T, Hjerpsted J, Raff M (2011) Palm olein increases plasma cholesterol moderately compared with olive oil in healthy individuals. Am J Clin Nutr 94:1426-1432

30. Dobbins RL, Szczepaniak LS, Myhill J et al (2002) The composition of dietary fat directly influences glucose-stimulated insulin secretion in rats. Diabetes 51:1825-1833

31. Liu L, Li Y, Guan C et al (2010) Free fatty acid metabolic profile and biomarkers of isolated post-challenge diabetes and type 2 diabetes mellitus based on GC-MS and multivariate statistical analysis. J Chromatogr B Analyt Technol Biomed Life Sci 878:2817-2825

32. Roggli E, Britan A, Gattesco S et al (2010) Involvement of microRNAs in the cytotoxic effects exerted by proinflammatory cytokines on pancreatic beta-cells. Diabetes 59:978-986

33. Belgardt BF, Ahmed K, Spranger M et al (2015) The microRNA200 family regulates pancreatic beta cell survival in type 2 diabetes. Nat Med 21:619-627

34. Lovis P, Roggli E, Laybutt DR et al (2008) Alterations in microRNA expression contribute to fatty acid-induced pancreatic beta-cell dysfunction. Diabetes 57:2728-2736

35. Thorens B (2013) The required beta cell research for improving treatment of type 2 diabetes. J Intern Med 274(3):203-214

36. Plaisance V, Waeber G, Regazzi R, Abderrahmani A (2014) Role of microRNAs in islet beta-cell compensation and failure during diabetes. J Diabetes Res 2014:618652

37. Ji X, Wang Z, Geamanu A, Goja A, Sarkar FH, Gupta SV (2012) Delta-tocotrienol suppresses Notch-1 pathway by upregulating miR-34a in nonsmall cell lung cancer cells. Int $\mathrm{J}$ Cancer 131:2668-2677

38. Zhou Y, Xiong M, Niu J et al (2014) Secreted fibroblast-derived miR-34a induces tubular cell apoptosis in fibrotic kidney. J Cell Sci 127:4494-4506

39. Peng X, Shao J, Shen Y et al (2013) FAT10 protects cardiac myocytes against apoptosis. J Mol Cell Cardiol 59:1-10

40. Lin CJ, Gong HY, Tseng HC, Wang WL, Wu JL (2008) miR-122 targets an anti-apoptotic gene, Bcl-w, in human hepatocellular carcinoma cell lines. Biochem Biophys Res Commun 375:315-320

41. Petros AM, Olejniczak ET, Fesik SW (2004) Structural biology of the Bcl-2 family of proteins. Biochim Biophys Acta 1644:83-94

42. Wali JA, Masters SL, Thomas HE (2013) Linking metabolic abnormalities to apoptotic pathways in beta cells in type 2 diabetes. Cells 2:266-283

43. Gardner BM, Pincus D, Gotthardt K, Gallagher CM, Walter P (2013) Endoplasmic reticulum stress sensing in the unfolded protein response. Cold Spring Harb Perspect Biol 5:a013169

44. Roggli E, Gattesco S, Caille D et al (2012) Changes in microRNA expression contribute to pancreatic $\beta$-cell dysfunction in prediabetic NOD mice. Diabetes 61:1742-1751

45. Roggli E, Gattesco S, Pautz A, Regazzi R (2012) Involvement of the RNA-binding protein ARE/poly(U)-binding factor 1 (AUF1) in the cytotoxic effects of proinflammatory cytokines on pancreatic beta cells. Diabetologia 55:1699-1708 